\title{
Immunological localisation of $\beta$-thromboglobulin and platelet factor 4 in human megakaryocytes and platelets
}

\author{
KATHRYN M MCLAREN, DUNCAN S PEPPER
}

From the Department of Pathology, University of Edinburgh Medical School, Teviot Place, Edinburgh EH8 $9 A G$ and the Scottish National Blood Transfusion Service, 2 Forrest Road, Edinburgh EHI 2QN

SUMMARY Beta-thromboglobulin ( $\beta T G$ ) and platelet factor 4 (PF4) have been localised in megakaryocytes and platelets using immunofluorescence and immunoperoxidase techniques. These studies support the concept of synthesis of the proteins by parent megakaryocytes. By immunoelectron microscopy both proteins have been visualised in the $\alpha$ granule of the platelet and megakaryocyte, supporting functional studies of the cytoplasmic localisation of these proteins.

The light microscopic techniques may allow elucidation of the distribution and role of the megakaryocyte in the pulmonary circulation and, on a practical level, permit its identification and distinction from other multinucleate cells in extramedullary tissue.

Platelet factor 4 (PF4)' and beta-thromboglobulin $(\beta T G)^{2}$ are two of the proteins released from platelets as part of the release reaction.' Although in vitro chemical functions have been ascribed to bothantiheparin $^{3}$ and anticollagenase ${ }^{4}$ activity to PF4 and inhibition of prostacyclin production ${ }^{5}$ to $\beta T G-$ evidence for a defined physiological or pathophysiological role is lacking. In order to gain an understanding of the functional role of the wide range of homologous platelet proteins and their role in platelet-tissue interaction, their site of synthesis and cytoplasmic localisation must be ascertained. A number of functional studies support the concept that the platelet proteins are stored in the $\alpha$ granules. ${ }^{6}{ }^{7} \mathrm{Up}$ to now, morphological studies have been confined to PF4 localisation, reporting its presence, by light microscopy, in megakaryocytes ${ }^{8}$ and platelets $^{89}$ by immunofluorescence techniques, and at ultrastructural level in platelets using an immunoferritin method. ${ }^{9}$ In this paper we report the localisation of $\beta T G$ and PF4 in megakaryocytes and platelets using a variety of immunological methods.

\section{Material and methods}

\section{LIGHT MICROSCOPY}

\section{Immunofluorescent localisation}

Smears of human bone marrow were used, both unfixed and after methanol fixation. The marrow

Accepted for publication 17 March 1982 was derived largely from cases of chronic myeloid leukaemia (CML), but included occasional patients without any histological marrow abnormality. No cases with known primary platelet dysfunction were used.

Indirect immunofluorescence (IF) microscopy using antisera to $\beta \mathrm{TG}^{2}$ and $\mathrm{PF} 4^{10}$ was used, with the antigen characterisation and determination of antibody specificity previously reported. "' Exposure to cytoplasmic content was achieved by prior saponification of membranes. Controls included normal rabbit serum as first antibody. In addition, the first step was replaced by incubation with a fraction of antibody absorbed with specific antigen. Sections were viewed with a Leitz Ortholux UV microscope.

\section{Immunoperoxidase localisation}

Blocks of lung and liver from cases of thrombocytosis and myelofibrosis were used for study of megakaryocytes in extramedullary tissue. Sections were selected which, by haematoxylin and eosin ( $\mathrm{H}$ and $\mathrm{E})$ staining, contained megakaryocytes lying in pulmonary alveolar capillary lumina or hepatic sinusoids.

Platelet-rich plasma, prepared using EDTA, theophylline and $\mathrm{PGE}_{1}{ }^{11}$ as anticoagulant, was centrifuged at $5000 \mathrm{rpm}$ for $40 \mathrm{~min}$ to form a pellet. All tissue was fixed in $4 \%$ buffered neutral formaldehyde embedded in paraffin wax and sections cut at $4 \mu \mathrm{m}$. The localisation technique used was the peroxidase antiperoxidase (PAP) method described by Burns. ${ }^{12}$ Controls comprised normal rabbit serum 
as first antibody. In addition, slides were incubated with both antisera after absorption by respective antigen.

\section{IMMUNOELECTRON MICROSCOPY \\ Marrow}

Human bone marrow aspirates, and platelet-rich plasma were fixed in a $1 \%$ paraformaldehyde, $1 \%$ glutaraldehyde mixture, in cacodylate buffer, embedded in Araldite resin, and sections cut at 50 $\mathrm{nm}$ on gold grids. For the marrow, areas containing megakaryocytes were first selected from $1 \mu \mathrm{m}$ sections stained by toluidine blue.

The localisation at electron microscopic level again used the PAP technique'2 with modification according to the work of Sternberger ${ }^{1.3}$ using a postfixation method. Innate peroxidase activity was blocked by exposure of grids to a $5 \%$ aqueous solution of hydrogen peroxide for $5 \mathrm{~min}$. Control grids were incubated with normal rabbit serum in place of $\beta$ TG and PF4 antisera. The grids were postfixed in $1 \%$ osmium for $15 \mathrm{~min}$ and examined with an AEI Corinth electron microscope.

\section{Results}

By light microscopy both $\beta \mathrm{TG}$ and PF4 were localised within megakaryocytes, using IF (Fig. 1) and PAP (Fig. 2) techniques. In the marrow staining was brightest (IF) in the larger cells, perhaps indicating a functional state correlating with stages of protein synthesis prior to platelet release. These cells showed obvious peripheral platelet release; the free platelets also exhibited bright cytoplasmic staining. There was no difference in IF staining between unfixed and methanol fixed sections.

The leukaemic tissue and normal marrow showed no difference in the megakaryocyte staining, though

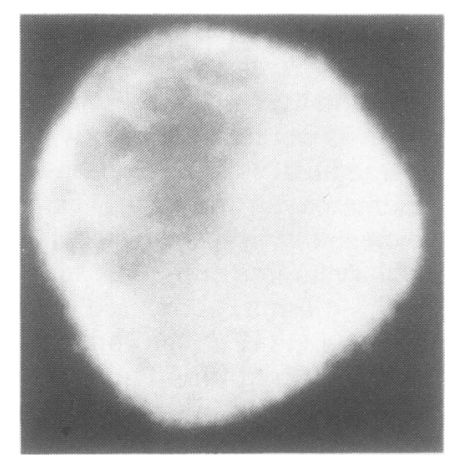

Fig. I Megakaryocyte in bone marrow. Citoplasmic fluorescence with PFt antisera $\times 1400$.

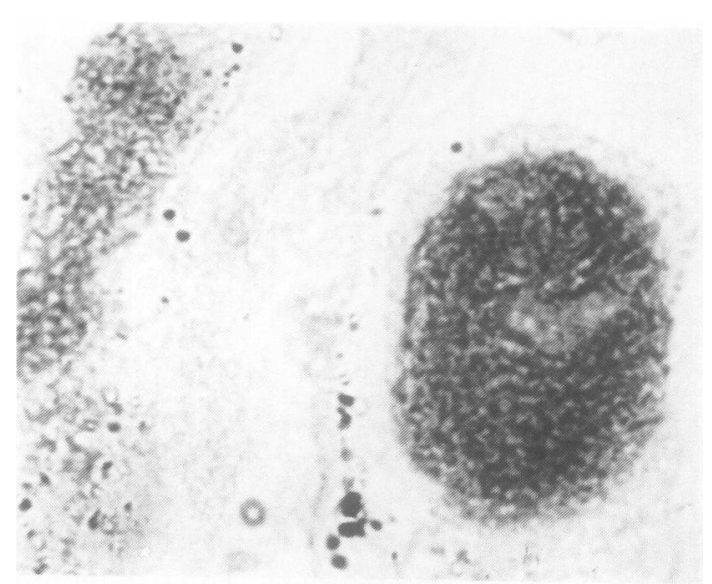

Fig. 2 Megakaryocyte in alveolar capillary. Immunoperoxidase reaction using $\beta T G$ antisera shows a cytoplasmic reaction product. Free platelets lie in a capillary to the left. PAP $\times 1250$.

there were. on the whole, greater numbers of megakaryocytes in cases of CML. Using the PAP technique a brown reaction product was present in many of the large, multinucleate cells in lung (Fig. 2) and liver which were recognised in $\mathrm{H}$ and $\mathrm{E}$ staine sections as megakaryocytes. Small tongues of peripheral cytoplasm, at times apparently separate from the cell body, may represent platelet release of a proplatelet extension. Occasional cells, morphologically conforming to typical irregular multinucleate megakaryocytes, failed to stain. Whether this represents a low protein content, undetectable by our techniques, a class or functional state of megakaryocyte or in fact other cell type(s), is not known. Some cells which, by $\mathrm{H}$ and $\mathrm{E}$ staining, may have been difficult to identify confidently as megakaryocytes, showed positive cytoplasmic staining. No positive staining was seen in controls using normal rabbit serum. Prior absorption of antisera by appropriate antigen also resulted in loss of megakaryocyte staining. By immunoelectron microscopy $\alpha$ granules were located throughout the cytoplasm of megakaryocytes. In those cells showing well formed demarcation membranes and obvious platelet formation, the granules were osmiophilic similar to those seen in platelets (vide infra). The presumed early stage of granule formation-that is, those forms associated with Golgi apparatus in megakaryocytes which lack a well formed demarcation membrane system, showed less of the osmiophilic aggregates. There was no obvious difference in complex number, osmiophilia or distribution on the granule between $\beta \mathrm{TG}$ and PF4 antisera. 


\section{PLATELET-RICH PLASMA}

The platelet pellet exhibited positive staining using antisera to $\beta$ TG and PF4 using the PAP technique. The resolution did not permit subcellular cytoplasmic localisation. Immunofluorescence studies revealed obvious platelet staining within bone marrow smears, particularly marked at the outer margin of megakaryocytes.

Using immunoelectron microscopy, the platelet granules exhibited dense, osmiophilic staining (Fig. 3). Aggregated, dense spherical deposits lay over the granules (Fig. 4); these resembled those described by Sternberger ${ }^{1.3}$ as representing PAP complex formation. Control sections showed only pale osmiophilia and scanty PAP complexes (Fig. 5). There was no consistent difference between $\beta$ TG and PF4 localisation, though the $\beta$ TG sections showed a more constant diffuse density of granule staining, with greater osmiophilia, than the anti-PF4 stained sections.

\section{Discussion}

The megakaryocyte origin of platelets and the ultrastructural identification of $\alpha$ granules within both megakaryocyte and platelet cytoplasm, with a relative paucity of synthetic apparatus-that is, endoplasmic reticulum and Golgi apparatus, in the platelet suggests that platelet granule proteins are indeed synthesised in the parent megakaryocyte. This concept has been supported by localisation studies of factor VIII ${ }^{14}$ and fibrinogen ${ }^{15}$ in the cytoplasm of both cells. In contrast, other factors, such as serotonin, are taken up by the platelet during circulation. ${ }^{16}$ The surface adsorption to platelets exhibited by a range of coagulation factors ${ }^{17}$ is also secondary, representing a

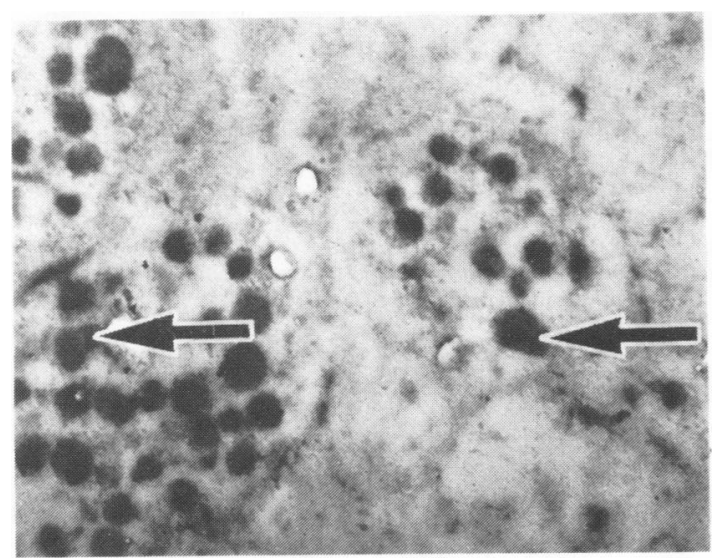

Fig. 3 Granules in platelets exhibit dense osmiophilia (arrow) $(\beta T G$ antiserum). PAP $\times 30000$.

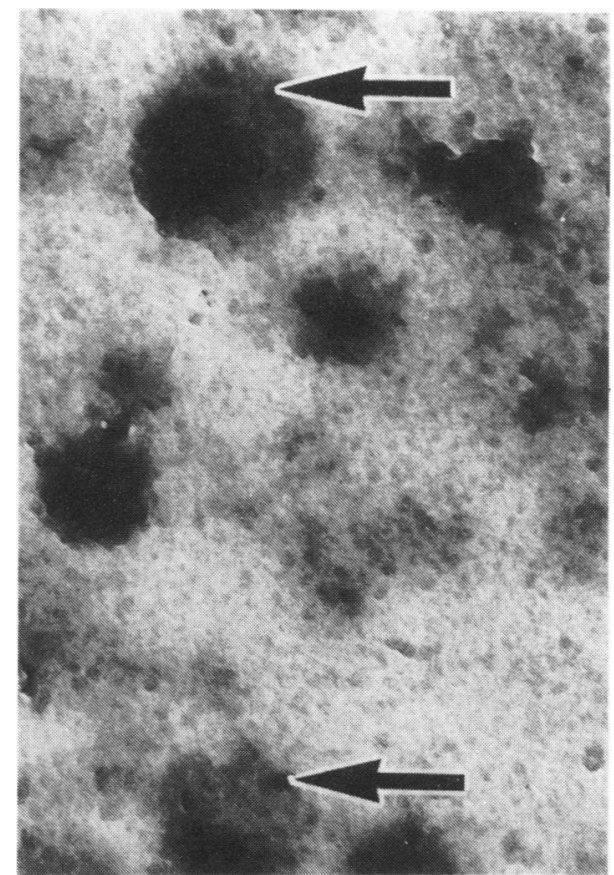

Fig. 4 At higher magnification, PAP complexes, represented by spherical deposits (arrows) lie in dense aggregates over the $\alpha$ granules. ( $\beta T G$ antiserum) $P A P \times 91000$.

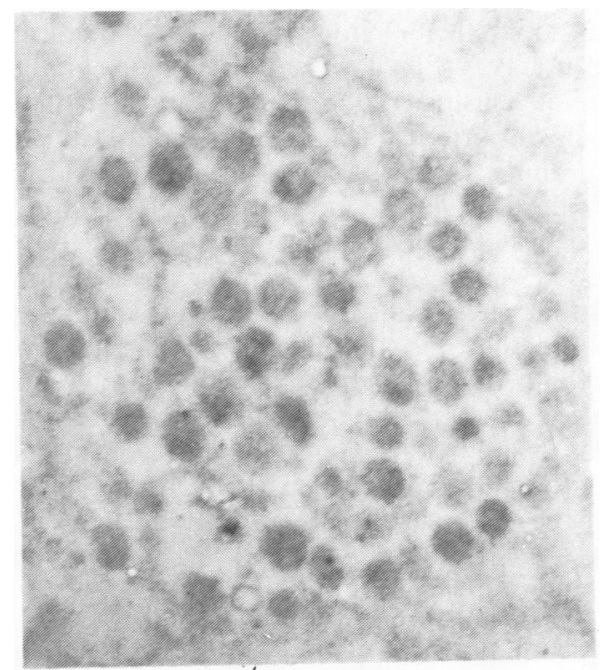

Fig. 5 Control section shows only pale osmiophilia of platelet granules. (At higher magnification only scanty complexes were seen.) $P A P \times 30000$. 
functional amplification mechanism in coagulation and not innate megakaryocyte synthesis. The present study supports the view that megakaryocytes synthesise $\beta$ TG and PF4. A similar study of PF4 localisation by immunofluorescence microscopy has been reported. ${ }^{7}$

We found that immunoperoxidase studies parallelled those of immunofluorescence. The former technique has a number of advantages. It can be performed on formalin-fixed tissue and can therefore be used in retrospective studies. It may therefore play a role in the identification of megakaryocytes in tissues outwith the bone marrow. There has been considerable controversy regarding the numbers, significance and indeed identification of these cells, especially in the pulmonary circulation. ${ }^{18}$ In the diagnostic area, there may be difficulty in distinguishing the multinucleate megakaryocyte from other types-for example, neoplastic cells. ${ }^{14}$ An appreciation of the nature of these cells may help us to establish their normal distribution in the lung, the means by which they may exhibit platelet release in a critical intravascular site without initiating disastrous coagulation mechanisms, and the role which pulmonary endothelial metabolism of vasoactive amines $^{211}$ and absence of prostacyclin degradation ${ }^{21}$ may play in this. In addition, the relationship of megakaryocytes to endothelial cell desquamation and syncytium formation, an appearance which may mimic multinucleate cells, may be resolved.

Several functional studies of platelet aggregation and the release reaction indicate that $\beta$ TG, PF4 (and other proteins showing homology such as plateletderived growth factor) are "secreted" at the time of $\alpha$ granule release. ${ }^{\circ} \mathrm{PF} 4$ localisation in the $\alpha$ granules of platelets, using an immunoferritin technique, has been reported. ${ }^{\star}$ The present study supports the $\alpha$ granule localisation of both $\beta$ TG and PF4 within megakaryocytes and platelets. There was no evidence by immunoelectron microscopy of diffuse cytoplasmic - that is, extragranular or membrane localisation. The platelets were, by simultaneous electron microscopic study, in an unstimulated state. The two proteins showed similar intragranular localisation, though the staining with antiserum to $\beta$ TG was usually more intense and diffuse within the granule than that using PF4 antiserum. This may support the concept of $\beta \mathrm{TG}$ as a matrix or packing protein since it may constitute the largest single component of the $\alpha$ granule. ${ }^{22}$

The short-lived nature of the platelet release reaction and the lack of knowledge regarding the duration of the subsequent role of the released products requires a combined morphological and functional approach to their study. Thus immunological localisation is a valuable correlative method in furthering our understanding of the functional properties of the platelet release proteins, their action and fate.

On a more practical level immunohistochemical markers for both megakaryocytes and platelets may be of value in the differentiation of giant cells and cytoplasmic masses within tissues and in evaluating their role in a number of physiological and pathological processes.

The work described in this paper was funded by a grant from the Wellcome Trust.

We acknowledge the help of $\mathrm{Dr}$ AC Parker. Department of Haematology and Dr C Prowse. Blood Transfusion Service for help in obtaining specimens. Thanks are due to $\mathrm{Mr} \mathrm{S}$ McKenzie and $\mathrm{Mr}$ RH Hogg for technical assistance.

\section{References}

Holmsen $H$. Biochemistry of the platelet release reaction in Biochemistry and Pharmacology of platelets. (iba Found Simp 1975:35:175-205

- Moore S. Pepper DS. Cash JD. The isolation and characterisation of a platelet-specific $\beta$-globulin ( $\beta$ thromboglobulin) and the detection of anti-urokinase and antiplasmin released from thrombin-aggregated washed human platelets. Biochem Biophys Acta 1975:379:36()-9.

'Levine SP. Wohl H. Human PF4: purification and characterization by affinity chromatography. J Biol Chem 1976. 251:324-8.

+ Hiti-Harper J. Wohl H. Harper E. PF4: an inhibitor ( collagenase. Science 1978:199:991-2.

s Hope W. Chesterman CN. Dusting CJ. Smith I. Morgan FJ: Martin TJ. Inhibition by $\beta$-thromboglobulin of prostacyclin $\left(\mathrm{PGI}_{2}\right)$ production in arterial endothelial cells. In: Thrombosis and haemostasis. Abstracts of the VIIth International Congress on Thrombosis and Haemostasis 1979:8.

- Day HJ. Subcellular localisation of platelet factor 3 and platelet factor 4. Scand J Haematol 1973:10:354

Witte LD. Kaplan KL. Nossel HL. Lages BA. Weiss HJ. Goodman De WS. Studies of the release from human platelets of the growth factor for cultured arterial smooth muscle cells. Circ Res 1978:42:4()2-9.

^ Ryo R. Proffitt RT. Poger ME. O’Bear R. Deuel TJ. Platelet factor 4 antigen in megakaryocytes. Thromb Res 198():17: $645-52$

" Ginsberg M. Taylor L. Painter R. Immunofluorescence and ultrastructural localisation of the secretory pathway of PF 4 (Abstract). Clin Res 1979;27:A295.

${ }^{1 t}$ McLaren KM. Holloway LJ. Pepper DS. Human platelet factor 4 and tissue mast cells. Thromb Res 1980:19:293-7.

"Ludlam CA. Cash JD. Studies on the liberation of (.)-thromboglobulin from human platelets in vitro. $\mathrm{Br} J$ Haematol 1976: 33:239-47.

12 Burns J. Background staining and sensitivity of the unlabelled antibody-enzyme (PAP) method. Histochemistri 1975:43: 291-4.

Sternberger LA. Immunocytochemistry 2nd ed. Prentice-Hall. 1978:130.

it Piovella F, Ascari E. Sitar GM. Malamani GD, Cattaneo G Magiulo E. Storti E. Immunofluorescent detection of factor VIII-related antigen in human platelets and megakaryocytes. Haemostasis 1974:3:288-95.

is Rabellino EM, Nachman RL. Williams N. Winchester RJ. Ross GD. Human megakaryocytes 1. Characterization of the 
membrane and cytoplasmic components of isolated marrow megakaryocytes. J Exp Med 1979;149:1273-87.

is Stacey RS. Uptake of 5-hydroxytryptamine by platelets. $\mathrm{Br} \mathrm{J}$ Pharmacol 1961:16:284-95.

- Walsh PN. Biggs R. The role of platelets in intrinsic factor $\mathrm{Xa}$ formation. Br J Haematol 1972:22:743-60).

- Pedersen NT. Occurrence of megakaryocytes in various vessels and their retention in the pulmonary capillaries in man. Scand $J$ Hatematol 1978:21:369-75.

1" Jackson JF. Histochemical identification of megakaryocytes from peripheral blood examined for tumour cells. Cancer 1962: 15:259-62. $\therefore$ Johnson AR. Erdos EG. Metabolism of vasoactive peptides by human endothelial cells in culture. J Clin Invest 1977:59:684-95.

$\because$ Moncada S. Korbut R. Bunting S. Vane JR. Prostacyclin is a circulating hormone. Nature 1978:273:767-8.

$\because$ Moore S. Pepper DS. Identification of a platelet specific release product: $\beta$-thromboglobulin. In: Gordon JL, ed. Platelet physiology Amsterdam: North-Holland. 1976:293.

Requests for reprints to: Dr Kathryn McLaren, Department of Pathology, University of Edinburgh Medical School, Teviot Place, Edinburgh EH8 9AG, Scotland. 